\title{
Impacto de la cultura en los resultados de la organización*
}

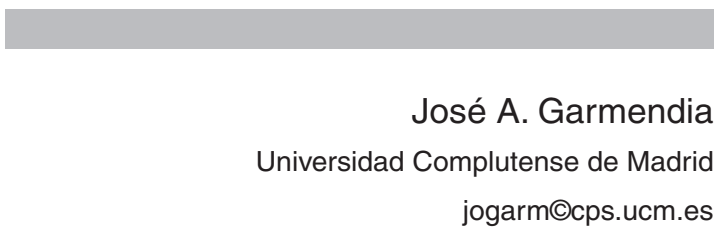

RESUMEN

Hablando de la organización es ya lugar común la afirmación del positivo impacto de una cultura fuerte en los resultados. La relación se ha refinado con el tiempo, de forma que la investigación ha concluido que aquélla es más sostenible si se introduce la variable flexibilidad. Es preciso aclarar que generalmente los resultados han sido considerados equivalentes a éxito económico e incluso a eficacia de la organización misma, de toda la organización. Este artículo, en cambio, trata de introducir complejidad definiendo la eficacia organizativa como la consecución de un sistema de valores (objetivos). Se parte de la hipótesis de que diferentes tipos de organización —definidos sobre la combinación de las variables éxito económico, fortaleza de la cultura, flexibilidad y adaptabilidad al entorno - tienden a producir diferentes perfiles de eficacia. El análisis de respuestas sobre una muestra de empresas del sector de seguros de vida y salud confirma nuestra hipótesis.

Palabras clave: Cultura Corporativa, Impacto de la Cultura Corporativa, Eficacia de la Organización.

\footnotetext{
* En este artículo se resume una investigación dirigida por el autor sobre el «Impacto de la cultura de la organización en los resultados de las compañías de seguros de vida y salud», financiado por el Ministerio de Ciencia y Tecnología. En el equipo figuraron destacadamente Alicia Kaufmann Hahn, Isabel de la Torre Prados y, de igual manera pero reforzando el diseño muestral y el análisis de datos, Francisco Fernández Fernández. En el trabajo de campo cabe mencionar igualmente a María Cruz López de Ayala y José L. López Jiménez, Asesor de Estrategias de Gestión de Personal.
}

Este artículo ha sido publicado, con ligeras modificaciones, en inglés por la revista Current Sociology, vol. 52, \# 6, pp. 10211038, y es ahora editado con permiso de Sage Publications en español por la REIS. 


\section{INTRODUCCIÓN}

Se ha convertido prácticamente en postulado que la cultura fuerte impacta positivamente en los resultados. Como todo tópico, éste contiene exageraciones y errores. Algo parecido ha ocurrido respecto de la proclamada relación entre la satisfacción y los resultados hasta que algunos (Lawler y Porter, 1967) dieron incluso la vuelta a la proposición. Concluyeron que si bien el grado de satisfacción laboral influye — por cierto, menos de lo que suele suponerse- en los resultados, la relación inversa es más cierta. De trasladar esta observación a la pareja cultura fuerte/éxito, sería procedente relativizar el peso de aquélla para no caer en algún tipo de determinismo culturalista. En efecto, los mismos resultados económicos influyen en el grado de fortaleza de la cultura. Por otra parte, el rendimiento depende de muchas variables, de forma que puede ser excelente incluso en empresas de cultura débil. Una de tales variables es la flexibilidad, que se ha tenido a menudo en cuenta (Kotter y Heskett, 1992; Denison, 1990; Soerensen, 2002) para afinar y atenuar aquella relación. Así, una cultura fuerte —entendida como «sistema de valores ampliamente extendidos en la organización e intensamente compartidos» (O'Reilly y Chatman, 1991: 493) — puede ser incluso negativa para el éxito si la cohesión que implica contribuye a la rutinización y el conformismo. En consecuencia, su ensimismamiento narcisista ignoraría los desafíos de un entorno crecientemente perturbador y competitivo con grave perjuicio en la cuenta de resultados.

La literatura referente a esta temática ha sido muy esclarecedora de la impronta de la cultura en los resultados. Sin embargo, adolece de ciertos inconvenientes que trataremos aquí de ir parcialmente superando. Unos se refieren al concepto mismo de cultura, otros al sesgo economicista en la definición del éxito y otros, finalmente, al manejo de un número relativamente modesto de variables, porque es preciso introducir más complejidad en los planteamientos, especialmente si se maneja un enfoque teórico más ambicioso.

El sector asegurador en España ha experimentado una fuerte reestructuración, que ha dado lugar a un proceso de concentración a través de fusiones, adquisiciones, alianzas estratégicas, etc. Como consecuencia de ello, en los últimos quince años ha desaparecido más del 15\% de las compañías y, aunque las aseguradoras han doblado su tamaño desde 1992, el mercado (especialmente en el ramo de Vida) presenta aún una elevada atomización. Recientemente, ha surgido con fuerza para el ramo de Vida el fenómeno de bancaseguros, que consigue fuertes sinergias entre dos ramas de actividad importantes.

Otro aspecto relevante en la evolución registrada ha sido la aparición de un cliente cada vez más exigente y que ha dado pie a la creación del Defensor del Asegurado. Éste debe atender, por otra parte, a una demanda creciente, dada la nueva composición del ahorro en los hogares españoles, el envejecimiento de la población, la incertidumbre sobre el sistema 
público de pensiones, la inestabilidad del mercado de trabajo, el incremento de planes de pensiones y seguros de vida y salud. El sector Vida ha venido experimentando en los últimos años incrementos superiores al 30\%, superando el año 2000 al negocio de no Vida debido parcialmente al éxito de los units linked.

En cuanto al ramo de Salud, son casi ocho millones de personas las que tienen alguna clase de seguro de salud, aunque sólo seis millones cuenten con cobertura completa. Por volumen de primas, el sector viene registrando los últimos años un crecimiento acumulativo en torno al $6 \%$. Los cambios producidos recientemente en la normativa fiscal han influido muy positivamente en el ramo. Tanto en éste como en el de Vida, las transformaciones han sido extraordinarias en un marco de importantes cambios económicos y sociales, que han supuesto desafíos organizativos, y, por supuesto, en la gestión y cambio de la cultura: entre otras razones, por la atención reclamada por un cliente más numeroso y exigente (cfr. Informes UNESPA e ICEA, fundamentalmente).

\section{ANTECEDENTES}

Desde las teorías propias de los recursos humanos se ha destacado la positiva relación entre capital humano (Becker, 1964), el compromiso con la organización y la eficacia de ésta (Argyris, 1964; Likert, 1961; Lawler, 1986; Siehl y Martin, 1988). En análogo sentido, se ha vinculado la participación con los resultados (Tannenbaum, 1968; Parker y Price, 1998). Sin embargo, es preciso no exagerar aquel vínculo (Wagner, 1994; Black y Gregersen, 1997) porque median multitud de factores que lo condicionan: entre otros, la calidad de las decisiones, que han podido ser desastrosas; el entorno y, por supuesto, aspectos simplemente económicos (Perrow, 1990: 107).

Ya he apuntado anteriormente al hecho de que el éxito es definido a menudo desde una perspectiva netamente económica. Daniel R. Denison (1990: 49) defiende esta postura «porque los indicadores correspondientes suponen una medida resumen del rendimiento de la organización como un todo... y son ampliamente considerados como tales por los que dirigen la empresa e invierten en ella». Consecuentemente, el citado autor relaciona rasgos culturales diversos (mejor dicho, de clima laboral) —por ejemplo, involvement (un índice combinado de tipo de organización del trabajo y toma de decisiones), «énfasis en los recursos humanos» y «coordinación entre unidades»- con dos indicadores de éxito o performance netamente conómicos: ratio beneficios sobre inversiones y ratio beneficios sobre ventas. Las conclusiones afirman un impacto positivo de aquellos rasgos en los resultados económicos al observar su evolución a lo largo de cinco años en una muestra de 34 empresas. 
J. P. Kotter y J. L. Heskett (1992) estudian la misma temática sobre una muestra de 207 empresas extraídas de 22 ramas de la industria en Estados Unidos. La correlación resultó ser aceptable, aunque modesta y, por ello, necesitada de explicar importantes salvedades: por ejemplo, el hecho de que empresas de cultura débil registraran éxito económico, mientras que otras de cultura fuerte fracasaran. Es decir, el vínculo cultura fuerte/éxito (Teoría I) es sostenible, pero queda corto en la explicación. Se refinó la anterior relación introduciendo la variable «ajuste al entorno» (Teoría II), y ello resultó ser más explicativo: en consecuencia, una cultura fuerte tendría un positivo impacto en los resultados si la misma se adaptara estratégicamente al entorno. Un corolario de este enfoque afirmaría incluso que un entorno predecible y relativamente estático requiere contenidos culturales menos adaptativos. Un paso más en la relativización del enfoque condujo a la formulación de la Teoría III, en el sentido de que una cultura fuerte producirá buenos resultados económicos no sólo si se adapta el entorno, sino si además se adelanta a sus cambios (contenidos proactivos). En cualquier caso, la fortaleza de la cultura es bienvenida en entornos altamente competitivos si éstos son tenidos en cuenta (Burt et al., 1994).

Afinando más la relación entre cultura fuerte y éxito económico, controlando el entorno, J. B. Soerensen (2002: 70) concluye así su investigación: «Los análisis de una muestra de empresas de una gran variedad de industrias evidencian que en entornos relativamente estables las organizaciones de cultura fuerte registran unos resultados menos variables. Sin embargo, en entornos volátiles los beneficios de aquella menor variabilidad declinan». Añade que esto se debe a la merma del aprendizaje de la organización frente a los desafíos internos o externos, precisamente por ser fuerte. Es decir, una cultura débil tendría siquiera una ventaja, la de no dejarse arrastrar tanto por rutinas aprendidas, mientras que una cultura fuerte podría sentirse cómoda y temerariamente autosuficiente ante situaciones inciertas. Con todo, se admite que el impacto de la cultura fuerte en los resultados es debido al impulso que la misma aporta al compromiso y motivación del personal, la facilitación del control social (O’Reilly y Chatman, 1996), así como la consistencia de normas y valores de la plantilla con la dirección, etc. (Gordon y DiTomaso, 1992).

El libro En busca de la excelencia (Peters y Waterman, 1984) concede un importante papel a la cultura corporativa -especialmente si sus valores pivotan en torno a la atención al cliente, la innovación y la proactividad/flexibilidad, la orientación a la acción y el desarrollo de los recursos humanos- en la promoción de la empresa llamada excelente y consecuentemente exitosa.

Finalmente, cabe mencionar una investigación más exigente con el concepto mismo de cultura. Más que el grado de fortaleza de la cultura, interesa a G. G. Gordon (1985) el 
tipo de cultura, considerada desde diversos ángulos o aspectos seleccionados -al menos aparentemente- sin criterio teórico previo alguno. A continuación relacionó el correspondiente perfil de rasgos con la rama de actividad, el carácter más o menos dinámico de la empresa e incluso con su éxito, concepto éste que no aparece claramente definido.

Cabe, pues, considerar algunos inconvenientes en el tratamiento del tema y que podrían resumirse del siguiente modo:

1. En cuanto al concepto de cultura, bien se lo ha dejado indeterminado limitándose a considerar su carácter fuerte o débil, bien se lo ha empobrecido haciéndolo equivaler a clima laboral, bien se lo ha desprovisto del requisito de totalidad en su definición, contándose con una batería ciertamente parca en valores y, en muchos casos, sin la debida justificación de que en ella «están todos los que son».

2. En cuanto al concepto de éxito o resultados (performance, organizational effectiveness) —no me refiero a otros resultados específicos, como el ajuste organización/personalidad (O'Reilly y Chatman, 1991) —, se lo limita a lo estrictamente económico. Sin embargo, esto resulta ser una merma conceptual porque una organización no produce sólo resultados económicos, sino, al mismo tiempo, mayor o menor solvencia, más o menos desarrollo de los recursos humanos, más o menos innovación, etc. Puede que la cuenta de resultados sea un triunfo, pero una derrota el escaso desarrollo de los recursos humanos, la pobre innovación en diseño, la modesta participación del personal en la toma de decisiones, la escasa devoción al cliente, etc. Además, lo definido como éxito por la dirección o los accionistas ha podido ser percibido como fracaso por un personal amenazado de despido o como fraude por parte del consumidor, etc. Tener en cuenta la totalidad de valores y de partes interesadas (stakeholders) es requisito de toda medida de la eficacia. De lo contrario, la relación cultura/éxito o la paralela cultura fuerte/éxito se encontraría empobrecida de antemano, bien se utilizara la perspectiva del «modelo natural de sistemas», enfatizando los aspectos de equilibrio de objetivos; bien la del «modelo racional o de consecución de metas», enfatizando el carácter instrumental de la organización; bien el «modelo de toma de decisiones», enfatizando el hecho de que las organizaciones procesan fundamentalmente información y decisiones; bien el «modelo ecológico poblacional», enfatizando los aspectos de control del entorno (Denison, 1990: 36).

Hay enfoques que han operado a la inversa: por ejemplo, seleccionando empresas marcadas por el éxito empresarial y extrayendo luego los factores de dicho éxito, sólo algunos de los cuales cabe considerarlos rasgos culturales. Es el caso de E. Bueno (1989), que hace equivaler éxito a competitividad y contempla los siguientes: 


\section{EXTERNOS}

Oportunidad de mercado

Demanda expansiva agregada

Descubrimientos tecnológicos

Nuevos recursos

Políticas de incentivación
INTERNOS

\author{
Espíritu innovador \\ Estilo directivo flexible y profesional \\ Misión clara y cultura integradora \\ Organización eficiente y adaptativa \\ Calidad del producto y de gestión \\ Innovación \\ Orientación al mercado y al cliente \\ Solvencia y autonomía financiera \\ Productividad y calidad informativa
}

Es preciso, pues, manejar una concepción más completa y abarcadora, tanto de los resultados como de la cultura misma. La mera contemplación de los principales elementos de la organización (constituencies: dirección, personal, cliente, accionistas, proveedores, la misma organización) exige definir los resultados como realizaciones más o menos logradas de los objetivos (valores) de la organización, tanto los económicos como los no estrictamente económicos. Por cierto, si estos últimos son descuidados acabará resintiéndose el mismo éxito económico y, en general, el rendimiento global.

\section{METODOLOGÍA}

La consecución de los objetivos derivados del proyecto de investigación nos condujo a las siguientes decisiones metodológicas:

\section{Selección de la muestra}

1. Teniendo en cuenta el informe previo de un actuario sobre el sector de empresas de seguros de vida y salud, consideramos el universo de las mismas como el conjunto jerárquicamente ordenado respecto del valor económico «éxito». Éste fue definido por combinación de indicadores objetivos, tales como incremento en los últimos cinco ejercicios del volumen de primas y de los beneficios respecto de la media del sector, solvencia e indicadores subjetivos varios de expertos y publicaciones especializadas. Se contó con prácticamente el total de empresas $(n=100)$ del sector, excluyéndose a algunas de muy pequeño tamaño.

2. Se depuró dicho universo, eliminándose:

- 15 empresas con fuerte imbricación, sobre todo de personal, con firmas del sector bancario; 
- 10 empresas por su escasa o nula dedicación a los seguros de vida y salud;

- 2 empresas por ser de estructura cooperativa y, al mismo tiempo, de escaso tamaño;

- 3 por ser de difícil contacto.

Por ello, el número de empresas consideradas equivaldría a 70 , ordenadas jerárquicamente según los indicadores mencionados.

3. Se establecieron tres grupos siguiendo el criterio del éxito económico. Esto es, un grupo de 20 empresas localizadas en los puestos más altos del ranking mencionado, un segundo grupo de 20 empresas -elegidas al azar entre las 30 ocupantes de posiciones intermedias-y, finalmente, un tercer grupo de 20 situado en la otra posición extrema.

4. A partir de esta clasificación se estableció un criterio de muestreo aleatorio simple y estratificado según los criterios anteriormente señalados, fijándose una muestra de 10 unidades por grupo.

5. Dado que el tipo de muestreo se puede considerar exploratorio, basado en casos-tipo según su ubicación respecto del ranking establecido en función del «éxito económico», no se puede referir stricto sensu un margen de error. Sin embargo, creemos que la representatividad estadística es razonable y aceptable para los propósitos de la investigación, interesada fundamentalmente en el contraste de tipos extremos. Hemos de señalar que del conjunto de la muestra establecida se consiguió recoger información directa de 27 empresas. Sin embargo, pudo obtenerse información del total de 30, al tratarse de una encuesta de tipo reputacional: es decir, cada empresa era preguntada por ella misma y las 29 restantes de la muestra*.

Se procedió a la aplicación de un cuestionario dirigido a tres directivos en cada empresa encuestada, siendo la respuesta producto de la previa discusión entre ellos.

\section{Definiciones e indicadores}

Se respetó el juicio de los directivos, en número de tres, en torno a la definición de la cultura fuerte o débil y del éxito, aunque en la introducción de la pregunta se insinuó el significado

\footnotetext{
* Las 30 organizaciones fueron las siguientes: Adeslas, Alico, Allianz, ARAG, Asisa, Atlántico Vida, Axa Seguros, Biharko, Cahispa, Cardif, Caser, Catalana Occidente, Génesis, Hércules Salud, La Boreal Médica, Lepanto, Mapfre, Musini, National Nederlanden, Pelayo Mondiale, Plus Ultra, Previsión Sanitaria Nacional, Prosperity, Reale, Sanitas, Seguros El Corte Inglés, Seguros Vitalicio, Skandia, Winterthur, Zurich España.
} 
ya consagrado del mismo concepto de cultura fuerte o débil. Se entiende que ellos poseen un conocimiento bastante aceptable sobre la situación de las otras empresas del sector en aquellas cuestiones. Cuando no había coincidencia se procuró llegar a un consenso y sólo en muy pocos casos fue necesaria una votación. Así, pues, interesaba fundamentalmente captar la imagen que las distintas organizaciones proyectan dentro del sector: por ejemplo, respecto del éxito. Este procedimiento ya ha sido aplicado por otros autores, sobre todo a partir de J. Woodward (1965), en su medida del éxito económico de empresas en función de las varias respuestas sobre una escala de tres posiciones: por encima, por debajo y en torno a la media del sector. Se les insistió que el éxito a lo largo de los últimos cinco ejercicios no deberían enfocarlo sólo desde la perspectiva económica (solvencia, tamaño, incremento de la cuota de mercado, del volumen de primas, de los resultados o de los beneficios sobre inversiones, etc.), recordándoles expresamente que la respuesta debería tomar en consideración, entre otros aspectos, la misma gestión, los juicios de expertos y de la prensa. Además, el encuestador conversó con los directivos sobre el lema de A. Schumacher «lo pequeño es hermoso" y sobre lo razonable de no equiparar automáticamente las realizaciones económicas con el éxito. Esta equiparación parece ser especialmente probable en el caso del tamaño. En efecto, investigaciones anteriores han registrado habitualmente una alta correlación, estadísticamente significativa, entre tamaño y éxito (Burt et al., 1994). El equipo avisó a los encuestados del peligro de contaminación de ambas variables, lo cual pudo contribuir a que la correlación registrada no fuera excesiva: $r$ de Pearson $0,4561(p<0,05)$, rho de Spearman $0,4581(p<0,05)$. El tamaño fue tratado en función del volumen de primas.

En relación con la definición, igualmente subjetiva, de «cultura fuerte» se procedió de modo análogo. Tan sólo se ofrecieron algunas guías en el preámbulo — gran sintonía del personal respecto de los objetivos y de la gestión general, escasa y débil presencia de subculturas disgregadoras, existencia de un estilo de organización y filosofía consagrado-, teniendo en cuenta enfoques clásicos de la literatura: «fortaleza» de la cultura como coherencia (Deal y Kennedy, 1982), como congruencia (Schall, 1983), como control internalizado (DiTomaso, 1987), como penetración de valores (Louis, 1985), etc. Por lo mismo, se prescindió de una elaboración a posteriori tan frecuentemente practicada, haciendo equivaler «fortaleza de la cultura» al inverso de la varianza en la distribución de respuestas. Como se ha indicado, el procedimiento fue considerado apropiado por dos razones: en primer lugar, se supone cierto conocimiento experto de los directivos; en segundo lugar, el estudio se interesó por la captación de la imagen de la organización, tal como era percibida por los encuestados.

Respecto del concepto de cultura, sus 15 rasgos considerados obedecen al propósito de abarcar todos los valores relevantes siguiendo enfoques anteriores inspirados en la literatura correspondiente: 
- solvencia, devoción al cliente, calidad/precio, búsqueda del beneficio, productividad, búsqueda de la expansión, desarrollo de los recursos humanos, participación del personal, remuneración del personal, liderazgo eficaz, importancia de las normas, importancia de la jerarquía, ética empresarial, flexibilidad/adaptabilidad, voluntad de innovación/proactividad.

Se tuvo especialmente en cuenta:

1. Una previa investigación del autor (Garmendia, 1992), que deriva aquéllos de la literatura clásica de los valores, específicamente de A. Maslow (1959), F. Herzberg (1959) y F. Parra Luna (1993).

2. El cuadro de orientaciones básicas (G. Hofstede, 1990, y otros cuadros habituales en el autor) en el estudio de la cultura de las organizaciones, así como la ya clásica tipología de P. Blau y W. R. Scott (1962) sobre la base del principio de cui bono.

\section{Una discusión en el seno del equipo investigador}

El propósito ha sido captar la cultura corporativa y la correspondiente eficacia como imagen, la que cada organización tiene de sí misma junto con las que otras organizaciones del sector han forjado sobre ella. Ciertamente, la imagen es también realidad y su gestión indudablemente necesaria si habremos de recordar el clásico postulado de W. I. Thomas: «Si los individuos definen las situaciones como reales, éstas son reales en sus consecuencias».

\section{Perfiles e hipótesis}

Con el fin de captar las prioridades de los rasgos culturales o valores - es decir, el sistema de los mismos como jerarquía - se formularon preguntas encaminadas a anclar la respuesta entre los extremos «muy característico» y «nada característico», siguiendo el procedimiento del Q-sort profile (Block, 1978), aplicado especialmente por Ch. A. O'Reilly y otros (1991). Así, los 15 indicadores (rasgos) señalados serían ubicados por los encuestados en cinco categorías según la pauta

\section{$2-3-5-3-2$}

Dicha pauta va de los 2 rasgos juzgados como los más característicos de la organización, en un extremo, a los 2 menos característicos de la misma en el otro. Continúa la ubicación 
desde los 3 siguientes rasgos juzgados como los más característicos a los 3 siguientes juzgados como menos característicos. La posición central recoge los 5 restantes rasgos: es decir, aquellos que no hayan sido mencionados en ninguno de los sentidos.

Con el fin de captar las diferentes intensidades de tales valores -otra forma de apreciar el sistema de los mismos- se estableció una escala ordinal de 1 (el valor menos característico) a 5 (el valor más característico).

Uno de los propósitos de esta metodología era descubrir diferencias significativas en los perfiles culturales entre empresas de mayor y menor éxito respecto de los mencionados 15 rasgos. En un análisis más agregado se perseguía detectar tales diferencias entre diversos tipos. Éstos, en número de 8, fueron elaborados en función de tres variables básicas: fortaleza de la cultura, grado de éxito y grado de flexibilidad/proactividad (cuadro 1). (Nota: Las letras de las distintas casillas corresponden a las empresas cuyo anonimato respetamos.)

\section{CUADRO 1}

Tipología de empresas

CULTURA FUERTE CULTURA DÉBIL

\begin{tabular}{rrrrr} 
& + FLEX/PROACT & - FLEX/PROACT & + FLEX/PROACT & - FLEX/PROACT \\
\hline + ÉXITO & 1 & 2 & 3 & 4 \\
\hline - ÉXITO & $\mathrm{a}, \mathrm{b}, \mathrm{c}, \mathrm{d}, \mathrm{e}, \mathrm{f}, \mathrm{g}$ & $\mathrm{h}, \mathrm{i}, \mathrm{j}$ & $\mathrm{k}, \mathrm{l}, \mathrm{ll}$ & $\mathrm{m}, \mathrm{n}, \tilde{\mathrm{n}}$ \\
& 5 & 6 & 7 & 8 \\
& $\mathrm{o}, \mathrm{p}$ & $\mathrm{q}$ & $\mathrm{r}, \mathrm{s}, \mathrm{t}, \mathrm{u}$ & $\mathrm{v}, \mathrm{x}, \mathrm{y}, \mathrm{z}, \mathrm{A}, \mathrm{B}, \mathrm{C}$
\end{tabular}

Así, pues, la eficacia y/o performance vendría reflejada en las realizaciones de los 15 rasgos antes mencionados en los 8 tipos de empresa a través de los perfiles respectivos.

Tales perfiles son considerados equivalentes a resultados o eficacia global de la organización (organizational performance), incluyendo el grado de éxito. Así, pues, se trató de ir más allá de los hallazgos acumulados respecto de la eficacia de la organización o resultados, al ser éstos considerados desde la perspectiva global de la misma cultura como resultado, reflejado en perfiles. Por lo mismo, se trasciende otros enfoques basados fundamentalmente en los resultados económicos. 
Con ello se facilitó el manejo de hipótesis, unas previas y otras extraídas por serendipity, referidas a teorías o tipologías anteriores: por ejemplo, la clásica de los sistemas mecánico/cerrados y orgánico/abiertos (Burns y Stalker, 1961; Buckley, 1967), la más difundida de orientaciones de la acción (orientación al entorno, orientación al beneficio, orientación al crecimiento, orientación a la organización, orientación a los recursos humanos, etc.), el cuadro de características de la empresa «excelente» (Peters y Waterman, 1984): orientación a la acción, cierta desburocratización, devoción al cliente, obsesión por la cultura, sistema orgánico de gestión, especialización en lo propio, etc.

La presente investigación no se aparta mucho de las preocupaciones ni de las conclusiones de estudios anteriores conocidos y ya mencionados. Sin embargo, trata de introducir complejidad en el enfoque diferenciando internamente los conceptos de cultura y eficacia de la organización. Se estudian ambos con ayuda de la técnica de los perfiles a fin de detectar adecuadamente su condición de sistema de valores (objetivos). Las mismas hipótesis se plantean sobre la base de dicho enfoque:

1. Las organizaciones de éxito registran un perfil de resultados -eficacia de la organización-más próximos a la "excelencia» que el resto, al igual que a un sistema orgánico de gestión.

2. Los tipos extremos —definidos respectivamente por la máxima y la mínima presencia de una cultura fuerte, éxito y flexibilidad - registran perfiles de resultados que contrastan significativamente en análogo sentido a la hipótesis anterior.

3. La orientación al entorno es crucial en la relación entre fortaleza de la cultura y resultados. Dicha orientación se basa fundamentalmente en la "devoción al cliente» y la flexibilidad/proactividad de la organización.

\section{RESULTADOS}

La fortaleza de la cultura y el éxito registran una correlación —la media de correlaciones dentro de cada empresa- más bien modesta $(r: 0,254 ; p<0,005)$, de forma que se da el caso de empresas de cultura débil acompañadas de un mayor éxito en tanto que éste se encuentra ausente de algunas de cultura fuerte.

Con el fin de afinar la relación cultura/éxito se ubicaron las distintas empresas en los 8 tipos (cuadro 1) antes mencionados, dicotomizándolas en torno a las sucesivas medias de las variables grado de éxito y, a continuación, de fortaleza de la cultura y flexibilidad/proac- 
tividad. Las letras de las distintas casillas designan otras tantas empresas. Las organizaciones de mayor éxito (las ubicadas en la fila superior del cuadro 1) arrojaron un perfil medio de resultados - es decir, de puntuación en los 15 rasgos considerados-diferente a las de menor éxito: es decir, las ubicadas en la fila inferior del cuadro 1. En efecto, en aquéllas se registró una mayor orientación al entorno (adaptabilidad/flexibilidad, innovación/proactividad, solvencia, ética empresarial), mayor orientación al desarrollo de los recursos humanos (formación, autorrealización, participación y remuneración del personal, liderazgo eficaz), mayor orientación al cliente (calidad/precio), aunque menor devoción al cliente (cuadro 2): esta última circunstancia estaría compensada por la mejor ubicación de las empresas de mayor éxito en el factor 1 «Orientación al entorno», donde la mayor carga en el factor corresponde a la "devoción al cliente» (véase luego la descripción del análisis factorial), pero menor orientación a la propia organización (importancia de las normas y de la jerarquía: es decir, una mayor tendencia a la burocratización) e igualmente menor orientación al beneficio (en cuanto búsqueda del beneficio).

\section{CUADRO 2}

Comparativa empresas Éxito-No Éxito

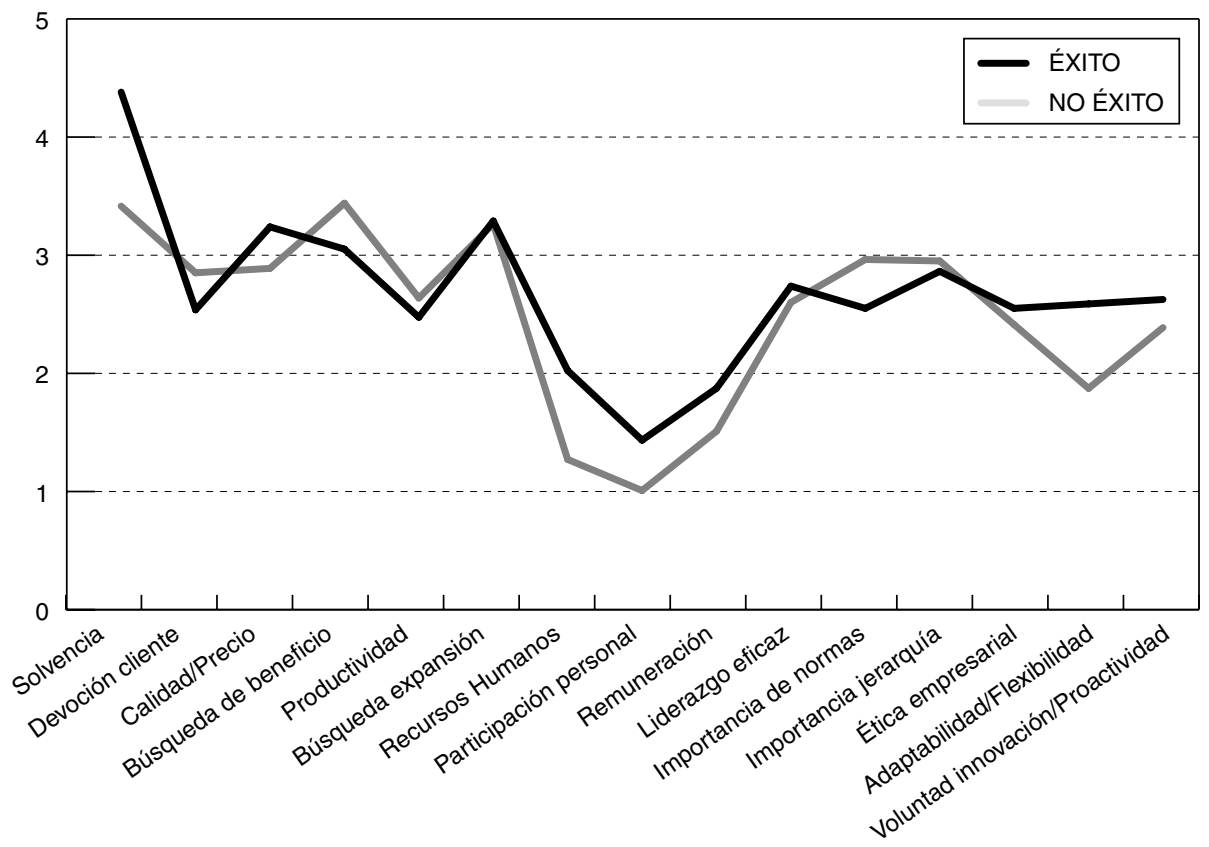

Rasgos culturales 
Nos aseguramos de la consistencia interna de las respuestas en los distintos items, obteniéndose un coeficiente de fiabilidad aceptable: Alpha de Cronbach de 0,78 a 0,85. Por otra parte, la diferencia entre curvas - aunque corta en cantidades numéricas, así como en los espacios del gráfico por haber manejado una escala reducida cuyos valores oscilaban entre 0 y 5 - es estadísticamente significativa en todos los rasgos: pruebas de significación $T<0,001$. Igual significación estadística se obtuvo en la diferencia de perfiles de tipos: por ejemplo, entre los perfiles de los dos tipos extremos 1 y (8), correspondientes a las casillas más densamente pobladas, cuyo contraste (cuadro 3 ) en cifras adelantamos.

CUADRO 3

Contraste tipos $1 /(8)$ en los principales rasgos

\begin{tabular}{cccccccccccccc} 
Solv & Dev & Cal/pr & Búsqb & Prod & Exp RR.HH. & Lid & Norm & Jer & Etic & Flex & Proact \\
\hline 4,23 & 3,12 & 3,45 & 3,56 & 3,05 & 3,63 & 2,34 & 3,15 & 3,22 & 3,34 & 3,05 & 2,83 & 2,97 \\
$(3,86)$ & $(2,89)$ & $(3,18)$ & $(3,62)$ & $(2,83)$ & $(3,61)$ & $(2,29)$ & $(2,77)$ & $(3,32)$ & $(3,44)$ & $(3,02)$ & $(2,57)$ & $(2,85)$
\end{tabular}

Como cabía esperar, ahora el contraste es mayor puesto que se han elaborado situaciones extremas: por una parte, la propia del Tipo 1, caracterizado por un mayor éxito, acompañado además de cultura fuerte y flexibilidad, y, por otra, la situación opuesta o Tipo 8 (cuadro 1). Así, las organizaciones Tipo 1, cabe destacar, aventajan a las de Tipo 8 -en cierto sentido, su opuesto- en orientación al cliente (solvencia, devoción al cliente, calidad/precio), orientación a los recursos humanos (desarrollo de los recursos humanos, liderazgo), orientación/ajuste al entorno (flexibilidad, adaptabilidad, proactividad, voluntad de innovación, ética empresarial) y orientación a la productividad. Sin embargo, están en desventaja respecto de la orientación a la organización (importancia de normas, jerarquía) y orientación al beneficio. Se ha incluido el rasgo «liderazgo eficaz» en la rúbrica orientación al desarrollo de los recursos humanos por varias razones obvias, una de las cuales es la correlación registrada entre ambos aspectos ( $r$ de Pearson: 353). En cuanto a la variable «ética empresarial», se la ha ubicado en el apartado de orientación al entorno teniendo en cuenta la carga institucional del concepto. Así, cabe considerar la ética como el «deber ser» de la organización o la armonía, más o menos lograda, entre los objetivos de ésta y su misión como mandato institucional o exigencia del entorno, sea ésta el imperativo moral del buen gobierno - por ejemplo, del consejo de administración—, la evitación del fraude al consumidor, etc.

Que las organizaciones de menor éxito coincidan con las del Tipo 8 en su mayor énfasis en la búsqueda del beneficio es digno de tenerse en cuenta. Dado, al mismo tiempo, su menor 
énfasis en el desarrollo de los recursos humanos, es razonable relacionar este hecho con aquella coincidencia. Es una reflexión razonable desde la teoría del capital humano. Cabe afirmar, pues, que el Tipo 1 responde más fielmente a los sistemas orgánicos de gestión, a las llamadas empresas excelentes y, en análogo sentido, a las organizaciones orientadas más por productos que por funciones y procedimientos. En parecido sentido, se han detectado diferencias en las realizaciones de valores entre empresas de sectores más y menos dinámicos. Se descubrió además que, dentro de cada uno de dichos sectores, las más adaptables al entorno eran, al mismo tiempo, las más rentables (Gordon y DiTomaso, 1992: 786).

En consecuencia, el contraste de resultados entre las empresas de mayor y las de menor éxito es semejante al registrado entre los tipos extremos 1 y 8 de las mismas. Tales resultados están situados en un continuum que va de las empresas de éxito, flexibles y de cultura fuerte, a las otras de menor éxito, poco flexibles y de cultura débil. Dicho contraste es el propio de los sistemas orgánicos frente a los sistemas mecánicos, acercándose los primeros a las definiciones convencionales de la «empresa excelente».

Estas conclusiones verificarían las dos primeras hipótesis antes establecidas.

Finalmente, partiendo de la matriz de correlaciones se procedió a un análisis factorial con rotación varimax, extrayéndose cinco factores, que explican un $72,796 \%$ de la varianza:

Factor 1. AJUSTE AL ENTORNO

0,741 Devoción al cliente

0,736 Voluntad de innovación/Proactividad

0,703 Búsqueda de la expansión

0,601 Adaptabilidad/Flexibilidad

Factor 2. ÉTICA Y EFICACIA ECONÓMICA

0,759 Ética empresarial

0,730 Búsqueda del beneficio

0,604 Solvencia

0,548 Productividad

Factor 3. EFICIENCIA Y CAPITAL HUMANO

0,853 Calidad/Precio

0,796 Desarrrollo de los RR.HH.

0,617 Liderazgo 


\section{Factor 4. ORIENTACIÓN A LA ORGANIZACIÓN}

0,542 Liderazgo

0,855 Importancia de las normas/reglas

0,781 Importancia de la jerarquía

\section{Factor 5. ORIENTACIÓN AL PERSONAL}

0,861 Remuneración del personal

0,736 Participación del personal

El factor Ajuste al entorno es el que más contribuye a la explicación de la varianza, con casi el $21 \%$. Al mismo tiempo, este factor y los cuatro restantes destacan en las organizaciones Tipo 1 frente a las de Tipo 8 en el sentido antes comentado (cuadro 3), como se aprecia en el cuadro 4.

CUADRO 4

\begin{tabular}{rrrrrr} 
Tipo 1 & factor_1 & factor_2 & factor_3 & factor 4 & factor 5 \\
\hline Media & 1,7823110E-02 & $2,227604 \mathrm{E}-02$ & $5,3230764 \mathrm{E}-03$ & $-4,939494 \mathrm{E}-02$ & $5,823655 \mathrm{E}-02$ \\
\cline { 2 - 5 } & 23 & 23 & 23 & 23 & 23 \\
\hline Desv. típ. & 1,0185696 & 1,0163680 & 1,0221270 & 0,9920868 & 0,9799790
\end{tabular}

\begin{tabular}{lrrrrr} 
Tipo 8 & factor_1 & factor_2 & factor_3 & factor 4 & factor 5 \\
\hline Media & $-0,2521879$ & $-0,1267157$ & $-0,3537893$ & $-0,1407225$ & 0,4794276 \\
\hline N & 8 & 8 & 8 & 8 & 8 \\
\hline Desv. típ. & 0,8292534 & 0,8958666 & 1,1024917 & 0,7844553 & 1,3974185
\end{tabular}

Análogamente, las empresas de mayor éxito puntúan sensiblemente más alto que las de menor éxito en el eje de dicho factor (cuadro 5). Sería una conclusión esperada de una de las características de la empresa excelente, la adaptabilidad como sinónimo de lo que 
Th. Peters y R. Waterman llaman «orientación a la acción» más «asunción de riesgos e innovación» (Gordon y DiTomaso, 1992: 789).

\section{CUADRO 5}

Peso de los factores según el éxito de las empresas

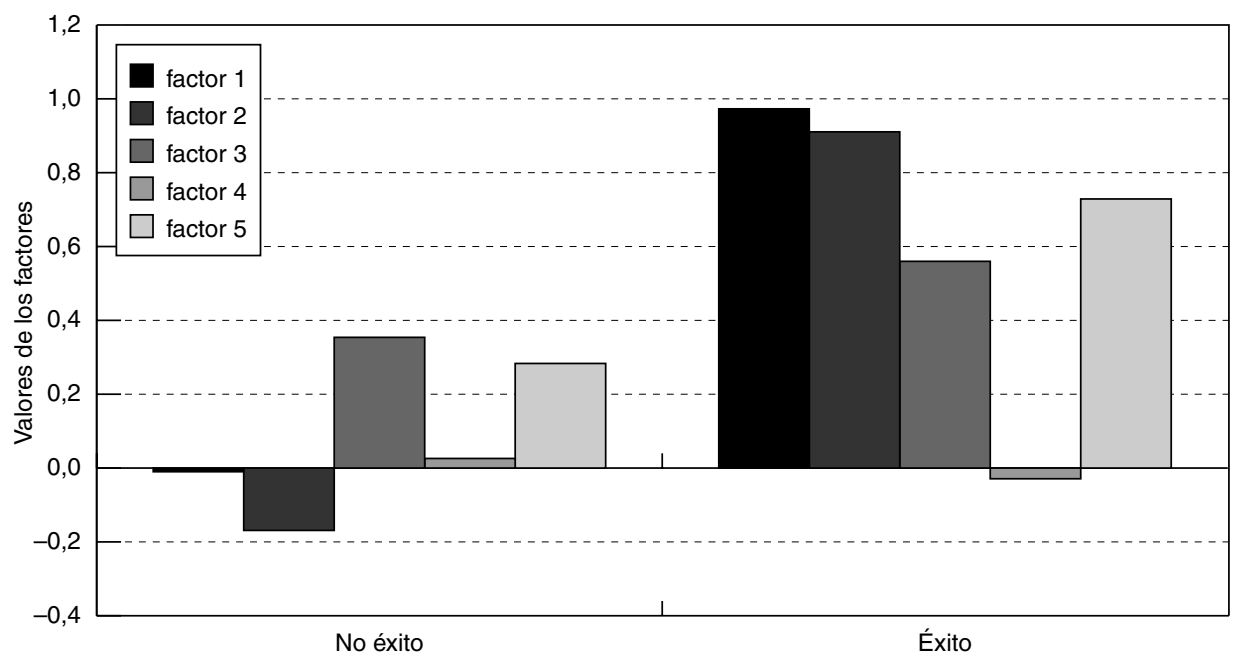

Cabe, pues, apuntar al hecho de que la orientación al entorno explica una parte importante de la varianza en la matriz de correlaciones de los rasgos culturales y que los tipos extremos de empresas, así como las de mayor y menor éxito, contrastan significativamente en dicha orientación. Esta circunstancia verificaría la última de las hipótesis, conforme con las meras exigencias de gestión de sistemas crecientemente abiertos al entorno, incluyendo el aumento de la competencia. En efecto, «que un sistema sea abierto significa que entra en intercambio con el entorno y que ese intercambio se convierte en factor esencial para la viabilidad del sistema» (Buckley, 1967: 83).

Sin embargo, es preciso limitar el alcance de la mencionada verificación de hipótesis debido a la existencia de importantes excepciones. Éstas pueden referirse a las organizaciones situadas a mitad del recorrido entre los dos tipos extremos - Tipo 1 y Tipo 8-. Así, una posición intermedia limitaría el alcance de las conclusiones, pensadas más bien para tipos ideales: es decir, configuraciones en las que intencionadamente se ha buscado la presen- 
cia exagerada de ciertos rasgos. Por otra parte, las diferencias de perfil entre los tipos extremos no son notables respecto de la intensidad con la que se da cada valor contrastado, incluso entre las distintas empresas en relación con los indicadores de éxito y fortaleza de la cultura (cuadro 6).

CUADRO 6

Estadísticos descriptivos de éxito y cultura fuerte

\begin{tabular}{lcccccr} 
& & & & & \multicolumn{2}{c}{ Estadísticos descriptivos } \\
\cline { 3 - 7 } & $N$ & Mínimo & Máximo & Media & Desv. típ. & Varianza \\
\hline Media de éxito & 24 & 4,84 & 6,83 & 5,9486 & 0,5344 & 0,286 \\
\hline Media cultura & 24 & 5,38 & 7,72 & 6,5710 & 0,5660 & 0,320
\end{tabular}

Las diferencias son menos notables todavía si se tiene en cuenta la prioridad de unos valores sobre otros: así, la prioridad concedida al valor «desarrollo de los recursos humanos» es la misma en los dos tipos extremos 1 y 8 , siendo en ambos casos relegado a la última posición o a la condición de rasgo menos característico; bien es verdad que en dicho valor hemos incluido también «remuneración del personal» y «participación del personal». Algo semejante ocurre respecto de los rasgos flexibilidad/adaptabilidad, innovación/proactividad, importancia de la jerarquía, productividad y devoción al cliente.

Así, pues, desde el punto de vista de la cultura corporativa, no hay diferencias sobresalientes entre los distintos tipos de organizaciones considerados, aunque sí podría haberlas entre algunas empresas específicas. Precisamente, la rareza y, por lo mismo, peculiaridad -en el sentido de difícilmente imitable - de una cultura suele comportar ventajas competitivas. Es una afirmación bastante consagrada en la literatura (Barney, 1991) que, para descollar en el mercado, una cultura necesitaría no sólo ser «buena», sino peculiar en el sector. Es también la conclusión a la que llegan E. Ogbonna y LI. C. Harris (2002: 812) en su investigación: «Se puede afirmar que las semejanzas culturales en muchos sectores pueden reducir el impacto que la cultura de la organización ejercería en el terreno de la competitividad..., siendo probable que sólo unas pocas empresas hagan uso estratégico de esa circunstancia». Habría que referir, pues, la modesta diferencia de perfiles de 1 y 8 a dicha circunstancia, así como admitir el escaso impacto de la cultura en el éxito de las organizaciones consideradas. 
Por otra parte, es preciso tener en cuenta dos excepciones notables referidas a los Tipos 4 (empresas de cultura débil y escasa flexibilidad pero con éxito: es decir, empresas designadas por las letras $m, n, \tilde{n}$ en cuadro 1) y Tipo 5 (empresas de cultura fuerte y gran flexibilidad pero con éxito menor: es decir, empresas designadas por las letras $o, p$ en cuadro 1). Cabe apuntar como explicación de tal «paradoja» a la misma teoría de la contingencia: es decir, en determinadas circunstancias (que habrían de ser investigadas) podría haber sido más adecuada para el éxito una menor flexibilidad y/o una cultura menos fuerte. Así, un exceso de flexibilidad podría haberse traducido en un liderazgo poco eficaz o en otras vicisitudes perjudiciales para el éxito, tales como una considerable impronta del comité de empresa en la marcha del negocio, o un exceso de fortaleza de la cultura podría haber generado en una patológica fidelidad a la misión establecida por los fundadores, etc. Indudablemente, procede aquí el estudio de casos, la referencia a la historia de la organización, el comportamiento del mercado y la competencia y, en general, ahondar en la «comprensión» weberiana para tratar de encontrar una explicación más convincente.

No procede afirmar incondicionalmente -eso quiere decir teoría contingente de la organización - la positiva relación cultura fuerte/éxito o la definitiva excelencia de un tipo sobre otro, como tampoco incondicionalmente la mayor bondad de un determinado estilo de dirección, pongamos por caso: «Nos inclinamos a decir que una organización lo ha hecho bien porque tiene un líder excepcional. Lo que generalmente queremos decir con esto es que han sido buenas las decisiones tomadas en relación con el tipo de producto o servicio, el control de calidad, las nuevas tecnologías y demás y no necesariamente que los líderes hayan exigido un gramo más de cooperación y motivación» (Perrow, 1990: 107).

Finalmente, habremos de indicar que en nuestra investigación no se han introducido variables explicativas, tales como el comportamiento del mercado o el tamaño de la organización, porque no se perseguía indagar posibles impactos de los mismos en los perfiles o eficacia global. Tampoco ha sido esto objetivo de trabajos anteriores en la literatura sobre esta temática y sí el manejo de tres variables fundamentales: éxito, cultura y flexibilidad.

\section{CONCLUSIONES}

Si bien las conclusiones no difieren sustancialmente de las ya obtenidas por otros autores, un enfoque más complejo y atento a la globalidad de la organización nos ha permitido redefinir de forma más exhaustiva la relación cultura y resultados. Así, se han definido los resultados o la eficacia de la organización en función de las realizaciones diferenciales en un total de 15 rasgos básicos, cuyo perfil se corresponde, al mismo tiempo, con la cultura corporativa en acción. Es un enfoque atento al hecho de la complejidad de las organizaciones: 
en consecuencia, lo que es visto como eficaz por los accionistas puede ser definido negativamente por el personal. Por otra parte, el éxito ha sido medido subjetivamente en respuestas reputacionales entre empresas y tratando de depurarlo de una visión exclusivamente económica o economicista. Se ha tratado, pues, de captar tanto la cultura corporativa como la eficacia de la organización como imagen, la percibida por la propia organización junto con la percibida por el resto del sector.

Se ha utilizado una metodología fundamentalmente cuantitativa, aunque el enfoque se ha aproximado al propio de los tipos ideales. Por lo mismo, hemos centrado la atención en el contraste de los opuestos, tanto del Tipo 1 versus Tipo 8 como de los de mayor éxito contra los de menor éxito en su conjunto. Es una estrategia encaminada a resaltar diferencias a partir de situaciones extremas. La exageración que, indudablemente, comporta la dicotomización sobre la presencia/ausencia de ciertos condicionamientos básicos — fortaleza de la cultura, éxito y flexibilidad-constituye un procedimiento útil incluso en la "comprensión». De todos modos, se impone la necesidad de completar el estudio con instrumentos de tipo cualitativo. Esto último constituye una nueva fase de la investigación, que resulta imprescindible por dos razones fundamentales:

- Comprender (verstehen) los casos desviados: por ejemplo, las paradojas que encierran los Tipos 4 (empresas de cultura débil y poco flexibles, pero acompañadas por el éxito) y 5 (empresas de cultura fuerte, gran flexibilidad, pero de menor éxito). Naturalmente, todo ello requiere además un estudio del contexto, fundamentalmente el referido a la rama misma de seguros de vida y salud, aunque, en nuestro caso, no se han ignorado desde un principio datos referidos al comportamiento económico del sector y de las mismas empresas investigadas. Tal ha sido también la mecánica de otros trabajos clásicos — por ejemplo, de Kotter y Heskett (1992) y Denison (1990) — y, más recientemente, de Ogbonna y Harris (2001). Estos últimos primaron la consideración de los sistemas culturales de dos organizaciones comparadas para luego añadir sentido mediante la referencia al entorno, el sector mismo de seguros de vida y salud. Esta referencia constituye una nueva fase de la investigación, que consistirá también en observación participante y entrevistas en profundidad.

- «Comprender» para explicar mejor los casos considerados «normales». Para ello se exige profundizar en el sentido de la cultura subjetivada o imágenes ya captadas en una primera fase. Esto último nos llevaría incluso a indagar en las raíces profundas de la cultura corporativa en la economía española: es decir, las presunciones básicas (Schein) o valores generales (Hofstede y Schwartz) (Ros, 2002).

Al igual que en investigaciones anteriores, podemos concluir que una cultura fuerte impacta positivamente en la eficacia organizativa (resultados), especialmente si aquélla se adapta al 
entorno e incluso es proactiva respecto del mismo. Ha de tenerse en cuenta, por otra parte, la modestia que en aquella relación introduce la perspectiva propia de la teoría de la contingencia organizativa, la cual ayudará a explicar los casos desviados. Finalmente, la misma modestia obliga a admitir que la eficacia de $A$ puede haber sido excelente pese a su débil cultura corporativa o escasa flexibilidad, o la de $B$ desastrosa pese a su cultura corporativa fuerte o su dinamismo y flexibilidad. La solución de la paradoja habría que encontrarla en otros factores, tales como las diferentes relaciones con el poder institucional, la mayor o menor calidad de las decisiones, el comportamiento del mercado, etc. Por supuesto, todo esto habrá de restar entusiasmo culturalista en la explicación de la misma eficacia de la organización.

El enfoque general aquí aplicado obedece fundamentalmente al espíritu de la Teoría del Capital Humano (Becker, 1964). Sin embargo, compartiendo algunas críticas, se ha tenido en cuenta no sólo la formación, sino otros intangibles, los propios de la empresa como sistema social: por lo mismo, nuestro enfoque teórico ha querido ser estrictamente sociológico. Obviamente, esto no excluye la consideración de indicadores económicos, pero exige la de muchos otros no estrictamente económicos.

\section{BIBLIOGRAFÍA}

ARGYRIS, C. (1964): Integrating the individual and the organization, Nueva York, John Wiley and Sons.

BECKER, G. (1964): Human capital: A theoretical and empirical analysis with special reference to education, Nueva York, Columbia University Press.

BLACK, S., y GREGERSEN, H. B. (1997): «Participative decision making: An integration of multiple decisions», Human Relations, vol. 50, 858 y ss.

BLAU, P., y SCOTT, W. R. (1962): Formal organizations, San Francisco, Chandler.

BLOCK, J. (1978): The Q-sort method in personality assessment and psychiatric research, Palo Alto, Consulting Psychologists Press.

BUCKLEY, W. (1967): La sociología y la moderna teoría de los sistemas, Buenos Aires, Amorrortu.

BURNS, T., y STALKER, G. M. (1961): The management of innovation, Londres, Tavistock.

BURT, R. S.; GABBAY, S. M.; HOLT, G., y MORAN, P. (1994): «Contingent organization as a network theory: The culture performance contingency function", Acta Sociologica, 37: 345-370.

DEAL, T., y KENNEDY, A. (1982): Corporate cultures: The rites and rituals of corporate life, Reading, Addison-Wesley. DENISON, D. R. (1990): Corporate culture and organizational effectiveness, Nueva York, John Wiley and Sons, p. 49. DiTOMASO, N.: «Symbolic media and social solidarity: the foundatios of corporate culture», Research in the Sociology of Organizations, 5: 105 y ss.

GARMENDIA, J. A. (1994): Tres culturas. Organización y recursos humanos, Madrid, ESIC.

GORDON, G. G. (1985): «The relationship of corporate culture to industry sector and corporate performance», en Kilmann et al., Gaining control of the corporate culture, San Francisco, Jossey-Bass, pp. 103-126. 
GORDON, G. G., y DiTOMASO, N. (1992): «Predicting corporate performance from organizational culture», Journal of Management Studies, 29: 783-799.

HERZBERG, F. (1959): The motivation to work, Nueva York, Wiley and Sons.

HOFSTEDE, G., et al. (1990): «Measuring organizational cultures: a qualitative and quantitative study across twenty cases», Administrative Science Quarterly, 35: 303.

KAUFMANN HAHN, Alicia (1999): Líder global: en la vida, en la empresa, Madrid, Univ. Alcalá.

KOTTER, J. P., y HESKETT, J. L. (1992): Corporate culture and performance, Nueva York, The Free Press.

MASLOW, A. (1959): «A theory of human motivation», Psychological Review, 56: 251-276.

LAWLER, E. L. (1986): High involvement management: Participative strategies for improving organizational performances, San Francisco, Jossey-Bass.

LAWLER, E. L., y PORTER L. W. (1967): «The effect of performance on job satisfaction», Industrial Relations, 7: 20-28.

LIKERT, R. (1961): New patterns of management, Nueva York, McGraw-Hill.

LOUIS, M. R. (1985): «An investigato'rs guide to work place culture», en Frost et al., Organizational culture, Beverly Hills, Sage.

MASLOW, A. (1959): «A theory of human motivation», Psychological Review, 56: 251-276.

OGBONNA, E., y HARRIS, L. L. (2002): «Organizational culture: a ten year, two-phase study of change in the UK food retailing sector», Journal of Management Studies 39 , vol. $39, n{ }^{\circ} 5$.

O'REILLY, Ch. A.; CHATMAN, J., y CALDWELL, D. F. (1991): «People and organizational culture: A profile comparison approach to assessing person-organization fit», Academy of Management Journal, vol. 34, n. ${ }^{\circ}$ 3, p. 493.

O'REILLY, Ch. A., y CHATMAN, J. (1996): «Culture as social control: Corporations, culture and commitment», en B. M. Staw y L. L. Cummings, Research in organizational behavior 18, Greenwich, JAI Press, pp. 157-200.

PARKER, L. E., y PRICE, R. H. (1998): «Empowered managers and empowered workers», Journal of Management Studies, $45: 85$ y ss.

PARRA LUNA, F. (1993): Balance integrado de gestión estratégica, Deusto, Bilbao.

PERROW, Ch. (1990): Sociología de las organizaciones, Madrid, McGraw-Hill, p. 107.

PETERS, Th. J., y WATERMAN, R. H. (1984): In search of excellence, Nueva York, Harper and Row.

ROS, M. (2002): «Los valores culturales y el desarrollo socioeconómico», REIS, 99: 9-35.

SCHALL, M. (1983): «A communications-rules approach to organizational culture», Administrative Science Quarterly, 28: 556 y ss.

SIEHL, C., y MARTIN, J. (1988): «Organizational culture: A key to financial performance», en B. Schneider, Organizational culture and climate, San Francisco, Jossey-Bass.

SOERENSEN, J. B. (2002): «The strength of corporate culture and the reliability of firm performance», Administrative Science Quarterly, 47: 70.

TANNENBAUM, A. S. (1968): Control in organizations, Nueva York, McGraw-Hill.

WAGNER III, J. A. (1994): «Participation's effects on performance and satisfaction», Academy of Management Review, 57: 312 .

WOODWARD, J. (1965): Industrial organization, Londres, Oxford University Press. 


\section{ABSTRACT}

Prevailing research claims that strong corporate cultures improve company performance. This paper addresses an unexamined implication of this argument by analyzing the effect of types of corporate cultures on company performance defined as the attainment of a complex system of values (goals). The approach goes beyond a concept of company performance merely as economic success, even if this implies negative consequences for some constituencies. I hypothesize that different organizational types defined as a combination of economic success, strength of corporate culture, flexibility and adaptability to the environment serve to obtain different effectiveness profiles. Results of the analysis of a sample of firms in the life and health insurance sector confirm our hypothesis.

Key words: Corporate Culture, Impact of Corporate Culture, Company Performance. 